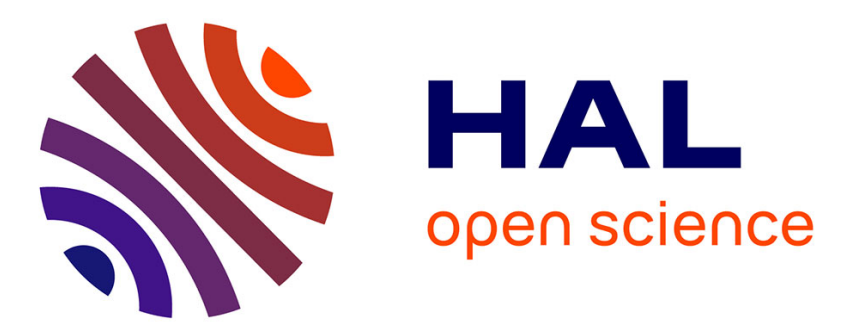

\title{
De l'idéal du critique au discernement du lecteur. Le traité préliminaire aux Jugements des savants d'Adrien Baillet (1685)
}

Emmanuelle Mortgat-Longuet

\section{- To cite this version:}

Emmanuelle Mortgat-Longuet. De l'idéal du critique au discernement du lecteur. Le traité préliminaire aux Jugements des savants d'Adrien Baillet (1685). Littératures classiques, 2015, 86 (1), pp.259280. 10.3917/licla1.086.0259 . hal-01407197

\section{HAL Id: hal-01407197 \\ https://hal.parisnanterre.fr/hal-01407197}

Submitted on 17 Feb 2021

HAL is a multi-disciplinary open access archive for the deposit and dissemination of scientific research documents, whether they are published or not. The documents may come from teaching and research institutions in France or abroad, or from public or private research centers.
L'archive ouverte pluridisciplinaire HAL, est destinée au dépôt et à la diffusion de documents scientifiques de niveau recherche, publiés ou non, émanant des établissements d'enseignement et de recherche français ou étrangers, des laboratoires publics ou privés. 


\section{Emmanue 11 e Mortgat-Longuet}

\section{De l'idéal du critique au discernement du lecteur. Le traité préliminaire aux Jugements des savants d'Adrien Baillet (1685)}

Lorsqu'en 1685 Adrien Baillet livre au public les premiers volumes de ses Jugements des savants, il les dote d'un important traité préliminaire intitulé «Des Jugemens sur les livres en général ${ }^{1} »$. Ce très long texte et l'«Avertissement » qui le précède entendent justifier son entreprise ${ }^{2}$, qui consiste à réunir l'ensemble des jugements portés sur chacun des auteurs "les plus considérables » dans tous les domaines de la production imprimée - même si, en l'occurrence, les derniers volumes prévus ne verront pas le jour ${ }^{3}$. Cette justification est d'autant plus importante pour la réflexion qui est la nôtre ici qu'elle consiste en une ample réflexion sur le rôle du critique, sur ce qui rend son intervention nécessaire, sur les différentes facettes de l'activité et des méthodes qui doivent être les siennes et sur tout ce qui peut les entraver. Ce texte témoigne donc, d'une manière unique et dense, d'une certaine conception de la critique des ouvrages à la fin du XVII e siècle, tant par la multitude des

1 Ce traité comporte près de six cents pages dans l'éd. in-12 des fugemens des sçavans sur les principaux ouvrages des auteurs, Paris, A. Dezallier, 1685-1686, 4 t., 9 vol. Nous le citerons désormais dans l'éd. posthume des fugemens des savans [...] revûs, corrigez, et augmentez par Mr. de la Monnoye, Amsterdam, Aux dépens de la Compagnie, 1725 [fac-sim. G. Olms, 1971], t. I, vol. 1 .

2 Bibliothécaire de François-Chrétien de Lamoignon depuis 1680 et précepteur de son fils, Baillet conçoit à l'origine ce projet pour guider les lectures et accroître les facultés de discernement de son élève, ainsi que pour distraire le magistrat. Il fut cependant conduit par son entourage à publier ses notes et son traité. Sur les circonstances de cette entreprise et sur les querelles provoquées par l'ouvrage, voir L. J. Wang, « La querelle des fugemens des sçavans d'Adrien Baillet (1685-1691) », XVII siècle, nº 84-85, 1969, p. 97-147.

3 Un plan de l'ouvrage fut livré au public en 1694, nous permettant de connaître l'architecture générale prévue par Baillet, mais seuls ont vu le jour les volumes concernant les imprimeurs, les critiques, les grammairiens, les traducteurs et les poètes. Baillet était sans doute bien lassé des polémiques suscitées par son ouvrage et déjà occupé à d'autres travaux, dont sa Vie de M. Des-Cartes (1691) et bientôt ses Vies des saints (1701-1703). 
questions abordées - procédant des réactions de son auteur aux nouvelles configurations de la vie littéraire ${ }^{4}$ - que par la cohérence, la fermeté et la richesse du propos. Et hormis l'article pionnier consacré par Françoise Waquet à ce traité en 1988, démontrant notamment tout l'intérêt de l'attention qu'y accorde Baillet à la « réception » des ouvrages, ainsi que les pages récentes dans lesquelles Jean-Marc Chatelain analyse l'actualisation qu'y fait le bibliothécaire du lieu commun de l' « excès des livres ${ }^{5}$ », il semble, à notre connaissance, que ces pages n'aient guère retenu l'attention des chercheurs, alors même que les diverses notices des fugements des savants, en revanche, continuent d'être utilisées, tout particulièrement dans les études de réception des écrivains ${ }^{6}$.

C'est donc apparemment plutôt par les réalisations concrètes qu'il offre que par les réflexions générales qui les introduisent que l'ouvrage de Baillet a suscité l'intérêt, et ce, en l'occurrence, dès l'origine - du moins lorsque les querelles qui ont vu le jour à l'occasion de sa publication se sont éteintes. En effet, comme Claude Faisant l'avait naguère remarqué, le XVIIIe siècle a très largement puisé la matière de ses jugements littéraires dans cette considérable " revue de la critique », en utilisant non seulement les citations faites par Baillet mais aussi ses propres commentaires ${ }^{7}$, ce qui confère incontestablement un rôle décisif à son ouvrage dans la formulation et la diffusion des évaluations des écrivains à cette époque. Il ne nous paraît pourtant pas possible que les caractéristiques du travail de Baillet, indéniablement à l'origine de son succès - nous pensons notamment au savoir, à la précision, à la sûreté et à la perspicacité dont il fait montre dans ses multiples notices -, puissent être disjointes de ses vues générales et des hautes

4 On trouve notamment, dans la seconde partie sur les «préjugés » qui nuisent à la juste perception des ouvrages, de nombreux développements sur la réputation des auteurs, leurs charges et dignités, leurs " engagements ", leur nation, leur humeur, leur âge, leurs modes et rythmes de production, les libelles diffamatoires, les abrégés et divers recueils, les livres anonymes, le titre des livres (question particulièrement étudiée par F. Waquet, voir n. 5), ainsi que leur taille, leur prix, leur débit, leurs récompenses, etc.

5 F. Waquet, «Pour une éthique de la réception. Les "Jugemens des livres en général" d'Adrien Baillet (1685) », XVII siècle, $\mathrm{n}^{\circ}$ 159, 1988, p. 157-174; J.-M. Chatelain, "L'excès des livres et le savoir bibliographique », Littératures classiques, ${ }^{\circ}$ 66, automne 2008, p. 145-160.

6 Quelques travaux ont entrepris d'examiner - incidemment ou non - la manière dont Baillet juge tel ou tel auteur. Voir notamment R. W. Tobin, "Racine, Sénèque et l'académie Lamoignon », dans M. Fumaroli (éd.), Critique et création littéraires en France au XVIIe siècle, Paris, Éd. du CNRS, 1977, p. 251-259 ; P. Dandrey, "Situation de Molière en 1683-1685 : diffusion, réception et influence de son œuvre dans la vie culturelle française », dans De la mort de Colbert à la révocation de l'Édit de Nantes : un monde nouveau?, Marseille, CMR 17, 1984, p. 377 392 ; C. Carlin, «Corneille devant les fugemens des savans : les éditions d'Oxford », XVII' siècle, n 225, oct.-déc. 2004, p. 677-684 ; E. Mortgat-Longuet, "Fabriques de Malherbe dans l'historiographie des lettres françaises (1630-1750) », dans L. Himy-Piéri et Ch. Liaroutzos (dir.), Pour des Malherbe, Caen, Presses Universitaires de Caen, 2008, p. 31-48. Cette liste n'est certainement pas exhaustive : nous prions ceux que nous pouvons y omettre de nous en excuser, et remercions d'avance ceux qui nous aideraient à la compléter.

7 G. Faisant, «Lieux communs de la critique classique et post-classique », Études françaises, t. XIII, n 1-2, avril 1977, p. 157. 
ambitions qu'il nourrit pour l'activité du critique : malgré l'écart inévitable qu'il y a entre un idéal et sa mise en pratique, malgré les opinions nécessairement contingentes du bibliothécaire et malgré ses évidentes limites personnelles, il est incontestable que ses convictions portent, accompagnent et déterminent la rédaction de l'ensemble de son ouvrage, et en éclairent la postérité.

En l'occurrence, ses convictions sur le rôle et sur l'activité du critique sont non seulement extrêmement précises et étendues mais encore fortes et singulières. En effet, même s'il est évident que Baillet est un héritier de la critique érudite et encyclopédique du siècle précédent, et même si l'on ne peut suspecter ce janséniste de la moindre complaisance à l'égard de l'épanouissement de la mondanité au sein de cette vie littéraire dont il est l'observateur, il n'en demeure pas moins qu'il témoigne également de mutations capitales de la pensée du XVII siècle, qui nourrissent et informent sa conception de la critique, l'éloignent en de nombreux points de celle de ses devanciers et lui permettent éventuellement de développer, à côté de positions plus attendues, une attention aiguë aux nouvelles missions qu'il confère, compte tenu des dernières évolutions du monde des lettres, à ceux qui jugent des ouvrages de l'esprit. Il s'agit donc pour nous d'essayer de saisir ces inflexions capitales que Baillet apporte à la fonction de critique - ce qui fonde et constitue l'importante promotion qu'il fait de sa nécessité et de ses modes d'intervention - et la manière dont il peut penser l'écart entre l'idéal qu'il édifie et les pratiques qu'il peut observer ou adopter. Nous allons donc chercher à comprendre à quel problème profond son propos entend répondre, puis nous considérerons les instruments conceptuels qu'il met en œuvre à cet effet, de manière à pouvoir examiner comment il est conduit à privilégier, parmi les rôles que le critique doit endosser, celui de la formation du lecteur.

\section{De la nécessité d'une médiation critique}

Des déséquilibres "à charge de la Républiques des lettres ${ }^{\circledR}$ "

En quels termes Baillet pose-t-il les problèmes qui le conduisent à rendre nécessaire l'intervention de l'activité critique ? À l'origine de son propos, on trouve effectivement la perception de divers déséquilibres et dysfonctionnements qui lui semblent caractériser la vie littéraire de son temps - perception dont nous allons tenter de restituer la logique sans tenir compte nécessairement de l'ordre dans lequel il fait ses remarques ni de la manière dont il peut ou non les enchaîner.

Il s'alarme de la multitude des livres, tout d'abord : en l'occurrence, c'est par ce lieu commun auquel il donne effectivement une «inflexion neuve ${ }^{9}$ » que

8 Expression est employée par Baillet à propos de la «masse confuse de la librairie » (op. cit., p. 40).

9 J.-M. Chatelain, art. cit., p. 153. 
Baillet débute son "Avertissement ». Effet néfaste de l'imprimerie ${ }^{10}$, la multiplication des ouvrages rend indistincts ceux qui constituent un réel apport à la pensée humaine : elle requiert ainsi une intervention dont il a été montré qu'elle ne consistait plus à limiter ou à contenir le nombre de livres dans des bibliothèques choisies ou universelles, mais à le contrôler par des productions susceptibles d'en régler l'usage ${ }^{11}$. C'est dans ce cadre que Baillet en appelle au « discernement ${ }^{12}$ » et qu'il promeut la figure du critique.

D'autre part, il prend acte d'une évolution qu'il nous semble fondamentalement penser comme une contradiction. D’un côté, Baillet constate la force extrême du jugement du public. Après Boileau, à qui il fait référence, il observe notamment que dans l'affaire du Cid tout le crédit de Richelieu ni toutes les raisons de l'Académie n'ont pu «effacer les impressions du Peuple, ni reformer les jugemens que les Particuliers ont faits d'une simple piece de Théatre ${ }^{13}$ » : il conclut donc à la disjonction, en cette matière, des "raisons » des instances étatiques et des décisions d'un public dont il souligne, en l'occurrence, une certaine autonomie de fait ${ }^{14}$. Aussi, s'il ne remet pas en cause le principe de la «liberté de juger ", Baillet s'inquiète en revanche de certaines de ses conséquences : il pose notamment la question de la légitimité de cette instance de juridiction ${ }^{15}$, ce public lui apparaissant particulièrement démuni, livré à lui-même, empêché par les faiblesses intrinsèques de ceux qui le composent - et pas seulement au théâtre ${ }^{16}$. Bien logiquement, l'érudit janséniste voit une des manifestations de cette faiblesse dans la part croissante accordée à la «manière » dans les jugements sur les écrits, signe d'un déséquilibre dans le partage à faire entre res et verba. Dénonçant ici un des effets, jugés néfastes, de la mondanité, Baillet déplore notamment le magistère des "ruelles des Dames » qui restreint la perception des ouvrages à leur forme, et celui du "peuple ", qui pèse particulièrement en ce qui concerne le théâtre : dans les deux cas, c'est le

10 A. Baillet, op. cit., p. XI (« Avertissement ») et 138 («Des Jugemens sur les livres »).

11 Nous nous inspirons ici des analyses de J.-M. Chatelain (art. cit., p. 153-160) auxquelles nous renvoyons.

12 A. Baillet, op. cit., par exemple p. XI («Avertissement»).

13 Ibid., p. 2 («Des Jugemens sur les livres »).

14 Sur la constitution, au XVII siècle, d'une entité juridique littéraire distincte de la simple juxtaposition des particuliers et déjà relativement autonome, ainsi que sur les débats concernant sa légitimité, voir H. Merlin, Public et littérature en France au XVIIe siècle, Paris, Les Belles Lettres, 1994.

15 Baillet déplore que les particuliers n'examinent pas si leur droit de juger librement est « naturel » ou « usurpé » (op. cit., p. 2).

16 Cette perception du public prend tout son relief dans le contexte des attaques portées par les jansénistes contre le théâtre, car Baillet s'en prend particulièrement au «peuple » qui entreprend de juger du genre dramatique. Ses propos dépassent cependant cette catégorie et visent l'ensemble des lecteurs en général, dans une perspective augustinienne : « notre cœur est un vase infecté qui peut corrompre tout ce qu'il reçoit » (op. cit., p. 10) écrit-il après Nicole («De la manière d'étudier chrétiennement », Essais de morale, éd. L. Thirouin, Paris, Puf, 1999, p. 250-252). 
manque de savoir qui occulte la juste perception des «matières » et fait triompher l'éclat trompeur des «vaines beautés du langage17 ». Cependant audelà même des faiblesses intrinsèques aux «fibres délicates » des femmes (ici Baillet s'appuie sur Malebranche) et de celles dont témoignent ceux qui n'ont aucune connaissance ${ }^{18}$, ce sont bien les faiblesses propres à tout homme qui oblitèrent sa juste perception des ouvrages de l'esprit : l'anthropologie augustinienne dans laquelle Baillet s'inscrit le conduit à souligner, dans ce domaine également, les méfaits de la «corruption du cœur de l'Homme » et de son amour-propre ${ }^{19}$. Ainsi, alors que ce public est désormais très puissant, il n'en reste pas moins livré aux irréductibles faiblesses qui menacent sa capacité à juger : pour pallier les méfaits de son amour-propre, pour prévenir ses carences et le prémunir contre ses «préjugés », il lui faut donc, lorsqu'il se préoccupe des ouvrages de l'esprit, un guide - une instance de médiation.

Enfin, tout montre que Baillet cherche à parer à la montée en puissance de la figure de l'écrivain. Il n'a de cesse en effet de souligner tout ce par quoi les auteurs sont susceptibles d'entraver le jugement du lecteur et qui témoigne de ce qu'il nomme leur « engagement » : leur vanité, leur humeur, leur condition, leur dépendance, leur soumission aux modes, leur utilisation des titres et des préfaces, leurs pillages, etc. Là encore, on mesure le poids de l'augustinisme dans les propos de Baillet : c'est que, dit-il en citant Nicole, la corruption de l'homme apparaît aussi dans ses livres, qui portent l'« impression insensible » des passions dans l'esprit de ceux qui les lisent ${ }^{20}$. Il n'est donc plus question ici d'un idéal héroïque de l'homme de lettres tel qu'il pouvait apparaître chez certains humanistes du siècle précédent. L'auteur lui aussi doit être conduit ou accompagné : il a besoin de «réformateurs ${ }^{21}$ ». Il est donc logique que dans ce contexte Baillet déclare abandonner les «éloges » qui ont pu être faits des hommes de lettres, et reporter à plus tard des propos sur leurs «vies ${ }^{22}$ ». Il estompe en quelque sorte la personne de l'écrivain au profit de ses écrits qui seuls fournissent matière à jugement, de manière, notamment, à ce que puisse être distingué ce qui est profitable à tous de ce qui ne l'est pas. Ce sont donc les productions de l'esprit humain qui doivent être au centre de l'activité critique bien plus que le «particulier » qui en est à l'origine : en cela Baillet rejoint les pratiques de certains de ses contemporains, comme Colletet, Sorel ou

17 A. Baillet, op. cit., p. 25-26, 8-10 et 20.

18 Ibid., p. 25-26.

19 Op. cit., p. 2.

20 Op. cit., p. 10. Voir P. Nicole, «De la manière d'étudier chrétiennement », Essais de morale, op. cit., p. 249-250.

21 A. Baillet, op. cit., p. 16.

22 Op. cit., p. XVI et XVIII («Avertissement»). Comme Baillet le dit lui-même, les précisions biographiques dans ses notices sont réduites au strict minimum, limitées le plus souvent aux dates de naissance et de mort. 
Fontenelle, dont les modes de pensée sont pourtant bien éloignés des siens ${ }^{23}$. Et l'on constate déjà aussi que Baillet isole, en ce qui le concerne ${ }^{24}$, l'activité de la critique des autres types de discours sur les écrivains.

Ainsi Baillet fait-il peser le soupçon sur le public et sur les auteurs. Pour prévenir les dangers que crée à ses yeux leur conjointe montée en puissance, il promeut le recours à une médiation, l'activité de la critique : elle est ainsi posée comme le seul antidote à ce qui, désormais susceptible d'échapper à la sphère d'influence du pouvoir et pourtant nécessairement menacé d'aveuglement, de fragilité et de corruption, serait sinon sans entrave.

\section{Des critiques faillibles}

L'autre versant du problème, cependant, consiste bien évidemment en ce que les critiques eux-mêmes n'échappent pas à la misère humaine. Ils sont également soumis à leurs passions, leurs intérêts, leurs liens de dépendance ou d'attachement. Baillet s'arrête, notamment, sur la divergence inévitable des critères évoqués dans leurs jugements ${ }^{25}$ qui, pourrions-nous penser, menaceraient du coup de s'annuler les uns les autres. Il n'en est assurément rien : certains sont pour lui objectivement contestables, comme il entend le montrer en récusant par exemple les critères du bon livre émis par l'abbé de Villars dans sa polémique contre Barbier d'Aucour ${ }^{26}$. Cette divergence ne conduit donc ici à aucun relativisme : elle résulte, en dernière instance, d'un problème de compétence et de valeur. G'est en effet sur ce terrain que Baillet prolonge les querelles, ici littéraires, entre jésuites et jansénistes, ce qui apparaît tout particulièrement dans la longue partie consacrée par lui aux «défauts » que le critique doit éviter ${ }^{27}$, nettement polémique : elle lui permet de s'en prendre, outre à l'abbé de Villars, à un certain nombre de jésuites en se reposant, pour des questions essentielles comme la précipitation dans les jugements, la pédanterie, la malignité ou l'amour-propre, sur des traités de Nicole et d'Arnauld, sur leur Logique, ou l'art de penser, ainsi que sur De la recherche de la vérité de Malebranche ${ }^{28}$. C'est qu'il s'agit d'imposer, sinon certains critiques

23 Sur cette question, nous nous permettons de renvoyer à notre article : « L'emploi du mot "vie" chez G. Colletet : de l'éloge de l'illustre à la critique du poète français », dans M. Jourde et J.-Gh. Monferran (dir.), Le Lexique métalittéraire (XVII'-XVII e siècles), Genève, Droz, 2006, p. 89106.

24 Cela n’empêche évidemment pas Baillet de classer dans la partie « Critiques historiques » des fugements des savants les auteurs de «bibliothèques", de recueils d'illustres et de divers catalogues.

25 A. Baillet, op. cit., p. 7.

26 Ibid., p. 8. Comme on le sait, Barbier d'Aucour qui avait attaqué Bouhours, était un proche des jansénistes. Et c'est contre lui que l'abbé de Villars avait entrepris de défendre Bouhours, en 1671, dans son Traité de la délicatesse.

27 Ces défauts sont la précipitation, la pédanterie, la chicanerie, la malignité, l'amour et la haine, et l'amour-propre (A. Baillet, op. cit., p. 30-40).

28 Voir notamment ibid., p. 31 et 35 ; et sur Malebranche, p. 39. 
précisément, du moins un certain type de critique : celle dont il pense que, tirant profit des meilleures réflexions concernant l'exercice du jugement, elle est à même de "faire distinguer les véritables beautés d'avec les fausses ${ }^{29}$ » - et sur laquelle nous allons bientôt revenir.

Il faut donc prendre acte de la fragilité du critique et de ce qui est susceptible d'oblitérer son jugement, de manière à établir les justes fondements de son art. Car les défaillances du critique ne sont pas seulement d'ordre intellectuel. En droite ligne augustinienne, Baillet estime que certaines tiennent «moins de l'esprit que de la corruption du cœur » : ainsi en va-t-il par exemple lorsqu'un critique flatte abusivement un "Grand » en vertu de sa « qualité » et de son « crédit ${ }^{30}$ », plutôt que du mérite de son ouvrage : les jugements émis du vivant de celui-ci resteront donc toujours suspects. De même, c'est en projetant sa propre malignité que le critique corrompu perçoit des défauts qui n'apparaîtraient pas à un cœur droit ${ }^{31}$, au risque d'être gouverné par son entière subjectivité. Aussi doit-il se défier de lui-même : sans toujours qu'il le sache, c'est par amour-propre qu'il loue l'auteur qu'il a souvent choisi en rapport avec lui ${ }^{32}$. Baillet s'efforce par là de soustraire l'activité de la critique à ce qui peut la dénaturer, l'amoindrir ou la perdre. Cette entreprise est d'autant moins facile que cette activité et ceux qui l'exercent jouissent dorénavant d'une mauvaise image. Il lui reste donc à tenter de la redresser.

\section{Une image à régénérer}

Baillet a en effet bien conscience qu'il doit mener l'offensive sur cette question. Comme on le sait ${ }^{33}$, le mot critique a déjà depuis un certain nombre d'années évolué dans un sens péjoratif, ce dont Baillet prend acte lorsqu’il

29 Ibid., p. 10. Baillet fait vraisemblablement référence sans le nommer, comme il le fait parfois, au texte de Nicole, Dissertatio de vera pulchritudine et adumbrata, placé en préface d'un Epigrammatum delectus publié par Port-Royal en 1659 : voir P. Nicole, La Vraie Beauté et son fantôme, trad. et éd. par B. Guion, Paris, Champion, 1996. Ce texte de Nicole avait connu un vif succès et un jésuite, le P. Vavasseur, l'avait combattu en 1669.

30 A. Baillet, op. cit., p. 54-55.

31 Ibid., p. 34-35. Ici Baillet adapte librement au cas du critique des propos de Nicole issus de son traité « Des jugements téméraires » (P. Nicole, Essais de morale, 8e éd., La Haye, A. Moetjens, 1700, t. I, p. 284).

32 A. Baillet, op. cit., p. 36-40. En prenant quelques précautions, Baillet vise tout particulièrement les religieux lorsqu'ils traitent d'auteurs de leur propre société, ainsi que les commentateurs. Et il finit par suivre Malebranche en reprenant presque tels quels de larges extraits de sa Recherche de la vérité (1. II, IIe partie, chap. 6, éd. G. Rodis-Lewis, Paris, Gallimard, « Bibliothèque de la Pléiade », 1979, p. 222-224).

33 Voir les travaux de J. Jehasse sur la critique et notamment ses chapitres sur l'évolution du terme : La Renaissance de la critique, Saint-Étienne, Publications de l'Université de Saint-Étienne, 1976, p. 663-678; Guez de Balzac et le génie romain (1597-1654), Saint-Étienne, Publications de l'Université de Saint-Étienne, 1977, p. 497-513. Et sur l'image négative du «censeur » ainsi que sur la naissance d'une «critique littéraire française », voir J.-M. Civardi, La Querelle du Cid (1637-1638), Paris, Champion, 2004, p. 199-222. 
explique qu'il lui a préféré, pour le titre de son ouvrage, celui de savant : «le terme de Critique semble avoir encore quelque chose d'odieux dans l'esprit de ceux qui ne sont pas entièrement guéris de leur prévention ${ }^{34}$ ». De fait, au moins deux vices sont désormais associés au critique par le public mondain, comme consubstantiels à sa fonction : la malignité et la pédanterie ${ }^{35}$. Raison supplémentaire pour que Baillet cherche à montrer qu'il les rejette, tant par ses attaques plus ou moins voilées contre tous ceux qui lui semblent faire montre, dans cette activité, de pédanterie ${ }^{36}$, que par sa dénonciation d'un critique chicaneur et obtus, uniquement préoccupé de nuire à un auteur ${ }^{37}$.

Quant à la critique elle-même, loin du bilan qu'on en peut faire aujourd'hui ${ }^{38}$, Baillet tend à la réduire à deux évolutions qui ont marqué les dernières décennies et qu'il cherche manifestement à contrecarrer. D’une part, au tournant des XVIe et XVII e siècles, la critique humaniste s'est recentrée sur la «philologie », et même si Baillet n'ignore pas le récent renouveau de l'érudition, il préfère mettre l'accent sur la perte ${ }^{39}$. On sait du reste que, dans ce mouvement, le commentaire du texte a été de plus en plus subordonné à l'établissement de celui-ci et que, dépouillé et réduit, il est dorénavant fréquemment rejeté dans les notes, à tel point que le goût du commentaire semble poursuivre sa carrière « en dehors du genre du commentaire ${ }^{40} »$. D'autre part, dans la voie ouverte par un Balzac et par les hommes de sa génération ${ }^{41}$,

34 A. Baillet, op. cit., p. XVII («Avertissement»). Voir aussi p. 31 (« Des Jugemens sur les livres ») : Baillet rend responsables les pédants d'avoir fait prendre ce «nom » de critique « en mauvaise part».

35 Voir J. Jehasse, Guez de Balzac et le génie romain, op. cit., p. 504-513.

36 Les exemples qu'il donne sont nombreux (op. cit., p. 31-32).

37 Ibid., p. 33-35.

38 On peut notamment citer les propos de R. Zuber qui juge, à propos de la critique au XVII" siècle, que " son discernement s'affirme et s'aiguise »; qu'elle " s'applique avec aisance à des œuvres en français, pour dégager en particulier les formes les plus insaisissables de l'écriture, celles de la prose » (Les Émerveillements de la raison, Paris, Klincksieck, 1997, p. 173).

39 « [C] ette literature universelle [...] faisoit anciennement la principale et la plus belle partie de la Grammaire, avant que les mauvais Grammairiens l'eussent deshonorée, et [...] obligée à changer son nom en celui de Philologie, [...] qui regardant essentiellement les mots de chaque Science, n'en traite les choses que rarement et par accident » (Fugemens des savans, page-titre, $2^{e}$ partie du livre sur les «Critiques », vol. I, t. II, p. N). Conjointement, Baillet estime que la critique semble avoir retrouvé « ces derniers tems » sa première « dignité », mais il ne donne aucun nom (op. cit., « Des jugemens sur les livres », p. 31).

40 Nous reprenons ici les analyses et les propos de J. Céard : « Les transformations du genre du commentaire », dans J. Lafond et A. Stegman (dir.), L'Automne de la Renaissance (1580-1630), Paris, Vrin, 1988, p. 101-115. D’autre part, à propos de l'évolution de la critique théâtrale au cours du siècle, J. Morel notait : « Aussi la critique théâtrale se glisse-t-elle partout, envahissant tous les autres genres, de la lettre familière au traité philosophique ou au sermon » («Les conditions de la critique théâtrale au XVII" siècle ", dans Actes de Las Vegas, PFSCL, « Biblio 17 », 1991, p. 13, cité par J.-M. Civardi, op. cit., p. 219).

41 Sur le rôle de transition joué par Balzac et sa génération dans cette évolution de la critique entre humanisme et mondanité, voir : J. Jehasse, Guez de Balzac et le génie romain, op. cit., p. 376- 
les «mondains » se sont emparés de l'activité critique au sens où ils se sont attribué, en vertu de leur esprit et de leur goût, le droit de juger eux-mêmes des ouvrages. Ainsi, pour le dire brièvement, la professionnalisation - l'« expertise »- de l'activité critique semble en passe de se réduire et laisse la porte ouverte, aux yeux de savants comme Baillet, à la promotion du « sentiment", au risque d'une perte éventuelle de l'objectivité des jugements. Baillet perçoit donc cette activité comme trop souvent ignorante de ses racines humanistes - les jugements des mondains le lui donnent à penser -, ou trop réduite à une philologie insuffisamment soucieuse des « choses » : dans ce cadre, il entend intervenir pour redonner à la critique des ouvrages, la jugeant en danger, tant objectivité que clairvoyance et pénétration, et pour la doter, l'estimant sans doute trop dispersée et trop poreuse, d'une certaine unité. Il lui faut donc établir à quelles conditions et avec quels nouveaux moyens conceptuels elle peut légitimement encadrer et orienter une saine et juste «République » des lettres.

\section{De nouveaux fondements à la critique?}

Une entreprise de conciliation

Comment donc pour sa part définit-il le bon critique? Les quelques adjectifs qu'il dissémine ici ou là pour le caractériser ne brillent guère, en eux-mêmes, par leur originalité : il est « sage », « savant », « habile » et « judicieux »; mais leur association peut malgré tout déjà donner quelques indications. À l'adjectif «savant » est désormais fréquemment associé l'adjectif «judicieux ${ }^{42}$ », qui relève des termes dont l'emploi, pour celui qui entend à leur moyen caractériser la « critique ", signale la volonté de « se distinguer à la fois des humanistes pédants et des modernes superficiels et désinvoltes ${ }^{43} »$ : Baillet tient à tempérer l'exigence du savoir, qu'il maintient fermement, par celle du jugement. Il peut du reste lui arriver de remplacer, aux côtés de l'adjectif «judicieux », le terme de «savant» par celui d'«habile ${ }^{44}$ », qui permet sans doute, même dans le contexte d'un écrit tissé d'érudition comme celui de Baillet, de tenir la « doctrine » à un peu plus grande distance.

Plus éclairant encore est l'ordre d'apparition des qualités du critique dans le chapitre que Baillet leur réserve. Évidemment, le savoir fait partie de celles qui lui sont nécessaires, même dans le cas, nous l'avons dit, de l'examen de la

402 ; R. Zuber, Les Émerveillements de la raison, p. 139-161 ; J.-L. Guez de Balzac, Euvres diverses (1644), éd. R. Zuber, Paris, Champion, 1995.

42 A. Baillet, op. cit., par ex. p. 10.

43 Propos de J. Jehasse dans Guez de Balzac et le génie romain, op. cit., p. 503.

44 A. Baillet, op. cit., par ex. p. 16 : les auteurs ont toujours eu le choix des personnes « habiles et judicieuses » auxquelles soumettre leur ouvrage avant sa publication. 
«manière ${ }^{45}$ ». Mais le savoir n’apparaît qu'en seconde place, après le «jugement ${ }^{46}$ ». Si la primauté du «jugement » est constamment évoquée dans l'ouvrage pour caractériser l'activité du bon critique, Baillet n'en offre pas une définition personnelle ou neuve en tant que telle. Cette première partie n'est fondamentalement qu'un assemblage d'extraits du traité de Nicole «De l'éducation d'un prince » et du premier discours de La Logique. Ou, plus exactement, elle n'est qu'une adaptation de ces extraits au cas singulier du critique à qui, donc, Baillet attribue les qualités que Nicole et Arnauld jugent nécessaires tant au pédagogue qu'à tout homme soucieux d'assurer sa pensée dans la recherche du vrai. Ainsi Baillet supprime-t-il quelques phrases ou parties de phrase de ses sources quand elles ne peuvent concerner le critique, ou au contraire ajoute-t-il certaines liaisons, propres à particulariser son propos. Par exemple, lors de la longue énumération qui, dans La Logique, énonce les caractéristiques de ces « esprits faux, qui n'ont presque aucun discernement de la vérité », Baillet, après en avoir reproduit mot à mot un certain nombre, remplace les propositions «qui ne font point de différence entre parler et parler ; ou qui ne jugent de la vérité des choses que par le ton de la voix ${ }^{47}$ » par «qui ne font point de difference entre écrire et écrire, ou qui ne jugent de la vérité des choses que par l'air que se donne un Auteur et par les maniéres de son stile ${ }^{48} »$ : il se moule ainsi sans réserve, en la particularisant, sur la pensée d'Arnauld et de Nicole. La qualité principale qu'exige l'activité critique est donc le «jugement », c'est-à-dire « le bon sens et la justesse de l'Esprit dans le discernement du vrai et du faux » (ce sont ici exactement les termes de la première phrase de La Logique qui eux-mêmes, comme on le sait, font référence à Descartes), et c'est bien ce jugement ${ }^{49}$ qui apprend aux « véritables Critiques »

à ne se pas éblouïr par un vain éclat de paroles vuides de sens; à ne se payer pas de mots ou de Principes obscurs, à ne se déterminer jamais dans leurs jugemens qu'ils n’ayent pénetré jusques au fond des choses traitées par un Auteur

et ainsi à « distinguer la délicatesse des choses d'avec celle des maniéres ${ }^{50}$ ». La cohérence et la force du cadre conceptuel d'Arnauld et de Nicole, d’inspiration

45 À ce sujet, contre les jugements des femmes et du peuple, il affirme avec force la nécessité d'une « science acquise » (ibid., p. 25).

46 Suivront l'intégrité, la douceur et l'indulgence, puis la modestie (ibid., p. 22-30).

47 A. Arnauld et P. Nicole, La Logique, ou l'art de penser, éd. D. Descotes, Paris, Champion, 2011, p. $130-131$.

48 A. Baillet, op. cit., p. 23. Il donne ici en note les références de ses sources, ce qu'il ne fait pas systématiquement.

49 Baillet précise ensuite ce jugement : « C'est le goût de l'esprit, c'est le discernement du vrai et du bon, c'est une délicatesse pour tout ce qu'il y a de faux et de mauvais » (ibid., p. 22). Ces propos sont très proches de ceux de Nicole sur le rôle du pédagogue dans « De l'éducation d'un prince » (Essais de morale, éd. L. Thirouin, op. cit., I, 19, p. 269).

50 Ici Baillet (op. cit., p. 23) reprend sans grand changement deux passages du traité «De l'éducation d'un prince » (loc. cit.) qu'il adapte au cas particulier du critique. 
cartésienne, permettent donc, aux yeux de Baillet, de doter la critique des ouvrages de nouveaux fondements et d'en faire un lieu privilégié d'exercice de la raison.

Cependant ces nouveaux fondements sont ici bien moins le point de départ de l'élaboration personnelle d'une théorie abstraite que les principes généraux à partir desquels il va pouvoir ensuite très concrètement spécifier cette activité critique. C'est notamment en associant à cette « exactitude de la Raison » dont le critique doit faire montre non seulement les plus hautes exigences éthiques, mais encore, très empiriquement, la nécessité d'un savoir très étendu que Baillet le dote d'une capacité à combattre tout "préjugé » en matière de production littéraire. On comprend donc en quoi il s'écarte sur ce point d'un Malebranche. Alors que celui-ci, à propos de l'étude, ne prône comme on le sait qu'une " science d'esprit », fondée sur des "vérités évidentes", et condamne toute « science de mémoire » qui ne relève « que des histoires et des faits ${ }^{51}$ » et dans laquelle il inclut ce qui ressort du « commentaire » et de la « critique ${ }^{52}$ », Baillet s'efforce quant à lui de souligner la pertinence et la nécessité de l'« exactitude de la raison » dans l'exercice de l'activité critique et de l'associer à l'exigence d'un savoir au moins théoriquement universel. Le bibliothécaire des Lamoignon entend ainsi renouveler la critique des ouvrages de l'esprit conformément à ceux qui, dorénavant, cherchent à appliquer l'esprit de méthode à d'autres domaines que les sciences exactes, comme l'histoire ou autres "lettres humaines", domaines que nous qualifierions aujourd'hui de «littéraires», ou encore comme les lettres sacrées ${ }^{53}$.

Une activité qui s'élargit

Dans cette entreprise de conciliation, Baillet profite des réflexions d'un Lamy qui venait de livrer au public la première édition de ses Entretiens sur les sciences ${ }^{54}$ - du reste, il cite cet ouvrage dès le début de son «Avertissement ${ }^{55}$ ». Dans une démarche qui synthétise tant son expérience de pédagogue que certaines conceptions de son confrère oratorien Malebranche ainsi que celles d'Arnauld et de Nicole dans leur Logique, Lamy condamne également toute érudition vaine. Mais il ne refuse pas l'acquisition d'un large savoir qui pourrait tendre à la formation de l'esprit et à l'accroissement des qualités morales et spirituelles. S'il place, outre la lecture des Écritures saintes, la logique et les mathématiques au

51 Malebranche, De la recherche de la vérité, 1. II, II ${ }^{\mathrm{e}}$ partie, chap. 4, éd. cit., p. 215.

52 Il conspue notamment « ces beaux esprits » qui font encore « leurs délices de la critique d'un mot, et du sentiment d'un auteur » (ibid., chap. 5, p. 218) et se moque de ces « généalogies ridicules d'opinions inutiles » qui sont « des sujets importants de critique aux savants » : voir ibid., p. 221 et p. 210-229 (chap. 3-6) ; 1. IV, chap. 7-8, p. 426-439. Baillet n'utilisera certains de ces propos de Malebranche - et sans toujours le citer - qu'afin de dénoncer le culte des anciens, ce qui en réduit considérablement la portée (op. cit., p. 44).

53 On peut penser notamment aux travaux de Richard Simon et de Jean Le Clerc.

54 B. Lamy, Entretiens sur les sciences [1683], éd. F. Girbal et P. Clair, Paris, Puf, 1966.

55 A. Baillet, op. cit., p. XI-XII (« Avertissement »). 
cœur de la formation de l'honnête homme chrétien, cela ne l'empêche pas de donner une part importante à des «sciences » telles que la grammaire, l'éloquence, les langues, la géographie et, tout particulièrement, l'histoire, si tant est qu'on les « prenne » bien et qu'on les étudie avec « méthode ». C'est dans ce contexte que la « critique » apparaît elle aussi comme une discipline positive. En effet Lamy promeut - comme Baillet va le faire à son tour moins de deux ans plus tard - la figure d'un « guide », notamment en matière de ce qu'il appelle encore ici (dans les deux premières éditions des Entretiens en 1683 et 1694) la " philologie 56 », dont " une des plus belles parties », estime-t-il, est « ce qu'on appelle la critique ${ }^{57} »$. À la différence d'un Malebranche, il plaide donc pour le recours aux commentateurs, comme à des « Maîtres qui nous font appercevoir ce que nous ne voyons pas » : c'est qu'il est «avantageux d'avoir un guide », c'est-à-dire

un habile Homme qui developpe les idées cachées et obscures de certains termes, qui fasse remarquer les delicatesses qui ne sont apperceües que de ceux qui ont le goût fin $[\ldots] .58$

Le terme de «critique » est ainsi, jusqu'en 1694 du moins ${ }^{59}$, employé par Lamy dans un sens théoriquement restreint - au masculin, celui d'un expert qui s'applique à la connaissance des textes des anciens -, mais on constate qu'il est déjà ici, au moins en ce qui concerne le substantif féminin ${ }^{60}$, en train de s'élargir, de se polir et de se moderniser, comme le montre encore cette restriction que Lamy préconise dans l'usage de ces critiques... au nom de la critique :

Ceux qui ont écrit des Biblioteques apprennent [...] quels sont les bons Critiques [...]. Mais il faut aussi de la critique et du discernement pour ne pas trop s'arrêter aux minuties, c'est à dire, aux observations de nulle importance que font les Critiques. ${ }^{61}$

Lamy fait donc figure, sur cette question, d'étape intermédiaire : si le domaine d'activité du critique apparaît encore, au moins dans l'édition de 1683, plus

56 «S]ous ce titre [de philologie] on comprenoit les Commentaires, les Paraphrases, les Glosses, les Remarques qui se font pour faire connoître la propre signification des termes dont les Anciens auteurs se servent "(B. Lamy, op. cit., p. 210, var., 1er état du texte [1683-1684] identique sur ce point à son $2^{\mathrm{e}}$ état en 1694 - les éditeurs ont retenu pour texte principal son $3^{\mathrm{e}}$ état, qui présente d'importants remaniements publiés en 1706).

57 Ibid., p. 211 (var., $1^{\text {er }}$ état du texte).

58 Ibid., p. 210 (var., 1 er état du texte).

59 À partir de 1706, dans le $3^{\text {e }}$ état du texte, la référence à la « philologie » disparaît, tandis que le terme critiquer connaît de plus nombreuses occurrences et qu'il est donné pour signifier « examiner avec jugement » : voir ibid., p. 214, texte principal.

60 Sur la différence des évolutions du substantif masculin et de l'adjectif, et du substantif féminin, voir notamment J. Jehasse, Guez de Balzac et le génie romain, op. cit., p. 498-499.

61 B. Lamy, op. cit., p. 211 (var., $1^{\text {er }}$ état du texte). 
étroit et plus spécialisé qu'il ne va l'être chez Baillet, il est quand même déjà apparenté à la figure globalement positive d'un "maître », capable de saisir les «manières » et le « caractère » d'un auteur - Baillet parlera du «stile » et des «maniéres d'écrire »- et capable de faire apercevoir au lecteur des « délicatesses » cachées, notamment grâce à ses facultés de discernement ${ }^{62}$ - on est dès lors bien loin de la simple figure du philologue.

Baillet profite donc de l'approfondissement que connaît alors la fonction de « critique » et notamment celui de la liaison, telle qu'elle peut être établie chez un Lamy, entre la pratique du commentaire et la mise en œuvre du discernement. Le bibliothécaire désigne effectivement par le terme de « critique » toute activité de jugement sur des textes ${ }^{63}$, quels qu'ils soient ${ }^{64}$, souhaitant la voir reposer désormais tant sur des connaissances expertes que sur une faculté de discernement. S'il donne encore le genre du commentaire pour une des parties de la « critique ${ }^{65}$, celle-ci n'est plus fondamentalement associée à l'activité du philologue. De cette manière, l'art de juger des ouvrages de l'esprit semble mis à l'abri du vice de pédanterie dont peuvent l'accuser les mondains. Pour autant, celui qui l'exerce n'est pas dispensé d'un savoir le plus vaste possible, si tant est que celui-ci ne le conduise pas à des jugements d'« autorité » et qu'il ne reste qu'un auxiliaire dans l'« examen » désormais « raisonné » des livres - élément supplémentaire pour que le guide soit d'une exceptionnelle qualité.

\section{De l'ethos du critique}

En effet, les exigences de Baillet en ce qui concerne le critique sont grandes, et ce d'autant plus qu'il inscrit résolument cet examen raisonné des livres dans un cadre éthique. Il a déjà été montré combien est présente cette dimension dans ses conceptions sur la production et la réception des livres ${ }^{66}$ et on la

62 «Lors qu'on a lû plusieurs fois un Auteur avec attention, on attrape ses manieres et le caractere qui luy est particulier, aprês quoy il n'est pas difficile de distinguer entre les Ouvrages qui portent son nom ce qui est de luy, ou ce qui n'en est pas [...]. Cette science se nomme Critique » (ibid., p. 211, var., 1 er état du texte). Dans son classement des critiques, Baillet, quant à lui, distinguera des philologues les «critiques historiques » dont l'activité s'applique dorénavant à tous les auteurs pour, notamment, « en faire le discernement, afin de ne point attribuer à l'un ce qui appartient à l'autre » et «juger de leur stile et de leurs maniéres d'écrire » (op. cit., page-titre, lère partie du livre sur les « Critiques », vol. I, t. II, p. A).

63 C'est du reste ce qu'indique le titre de son traité initial, « Des Jugemens sur les livres en général ».

64 L'art de juger, pour Baillet, se donne à voir tant dans les Sentiments de l'Académie sur le Cid que dans les Pères de l'Église, tant dans les jugements sur les auteurs anciens que dans ceux sur les modernes, tant dans les écrits des critiques humanistes que dans ceux d'un Segrais, d'un Marolles ou d'un Boileau, voire dans ceux des femmes - quitte à discréditer celles-ci : voir op. cit., p. 2-3, 5-6, 11-14, 19-22, 24-25, etc.

65 Ibid., p. 39.

66 Voir F. Waquet, « Pour une éthique de la réception », art. cit., notamment p. 163-166 et 171172. 
retrouve logiquement dans ses propos sur l'intervention du critique : il doit déciller les yeux des auteurs et des lecteurs, les défaire du piège des apparences, les prémunir, en l'occurrence, contre l'« éblouissement » des fausses beautés ${ }^{67}$. Il s'agit donc d'un véritable combat, comme le montre la reprise ici des traditionnels propos sur les vertus paradoxales du blâme et l'«heureuse violence » de la censure qui, pour peu qu'ils soient exercés civilement, sont source de grands progrès ${ }^{68}$. Cette "Critique contentieuse » est cependant particulièrement soumise à la passion et aux intérêts particuliers : celui qui l'exerce doit donc être en mesure de les tempérer en lui-même. Ainsi les qualités que Baillet attribue au critique, après l'exactitude du jugement et la science, nous semblent-elles jouer le rôle de "régulateurs» face à la menace que représentent ses propres inclinations. Ce seront notamment l'intégrité, cette " équité incorruptible ", dit-il en citant Bodin ; la douceur qui, jointe à la sévérité, permet de se tenir dans les bornes de la prudence et de la justice ; puis l'indulgence, qui fait avoir "divers égards à toutes les circonstances favorables qui peuvent excuser ou justifier un Ecrivain »; et enfin la modestie ${ }^{69}$ qui, estimet-il en s'appuyant sur saint Augustin, confère paradoxalement ascendant et autorité :

C'est elle qui gagne le cœur de toutes sortes de personnes. C'est elle qui établit la réputation d'un Critique, et qui lui attire sans violence l'estime des Lecteurs, et la confiance des Auteurs. ${ }^{70}$

Or, dans le passage de la correspondance de saint Augustin auquel Baillet fait référence, correspondance traduite du reste un an plus tôt par Philippe Goibaud Du Bois ${ }^{71}$, il ne s'agit évidemment pas de critique littéraire, mais d'une persuasion réussie à propos d'une question judiciaire. Macedonius, «vicaire d'Afrique ", dit avoir été convaincu de gracier des criminels grâce à la «modestie » que l'évêque d'Hippone a déployée dans sa lettre à ce sujet : il est question ici de l'effet de cette qualité dans le domaine de l'éloquence. Baillet ne fait donc que transposer à la critique cette disposition, la modestie qui, déjà évoquée par Cicéron ${ }^{72}$, est surtout développée par la tradition chrétienne de l'art oratoire, chez saint Augustin effectivement, mais encore tout particulièrement au cours du XVII siècle, notamment chez Bernard Lamy ${ }^{73}$.

67 A. Baillet, op. cit., p. 10, 18-19, 22-23, etc.

68 Ibid., p. 18-19.

69 Ibid., p. 26-30

70 Ibid., p. 30.

71 Les Lettres de saint Augustin, traduites en françois sur l'édition nouvelle des Peres Benedictins de la Congregation de S. Maur [par Ph. Goibaud Du Bois, annotation par S. Le Nain de Tillemont], Paris, J.-B. Coignard, 1684, t. II, lettres GLIV-CLV, p. 166-167 et 176-177.

72 Cicéron, De oratore, II, XLIII, 182.

73 B. Lamy, La Rhétorique ou l'Art de parler, éd. C. Noille-Clauzade, Paris, Champion, 1998, 1. V, chap. 11, p. 405-408. 
Cette transposition d'une qualité déterminante de l'ethos de l'orateur chrétien nous donne ainsi la mesure de la constitution, dans le texte de Baillet, d'un ethos du critique, chrétien en l'occurrence, seul susceptible de permettre finalement, au-delà de ses indispensables qualités intellectuelles, de lui gagner les cœurs.

Il se dessine donc progressivement une image très forte du critique, car son action dans le domaine des "productions de l'esprit » ${ }^{74}$ se joue, en dernière instance, sur le terrain des mœurs : elle requiert donc la plus large palette de dispositions personnelles ${ }^{75}$. Le personnage construit par Baillet apparaît finalement comme un personnage hors du commun, un être d'exception. Ainsi finit-il par affirmer que

pour composer un bon Critique, il faut faire l'assemblage de toutes les excellentes qualités dont quelques-unes suffisent séparément pour faire un habile homme dans les autres connoissances. Car il ne suffit pas à des Censeurs ou Critiques d'être doués de celles qui leur sont communes avec les Auteurs qu'ils soumettent à leur examen, il faut qu'ils fassent encore en eux-mêmes l'union de celles qui paroissent incompatibles dans les Personnes des autres Professions, ou dont la rencontre n'y est pas du moins absolument nécessaire. 76

Ge composé de toutes les qualités habituellement incompatibles dans un seul homme, et qui n'est pas sans évoquer la «perfection en tout genre » que Cicéron souhaitait pour l'orateur, montre que Baillet transfère ici au critique cette idée des personnalités « récapitulatives », comme on a pu le faire à la fin du XVIe siècle pour Ronsard avec le topos de l'« universalité de son esprit77». Cependant, si cet idéal est chez Baillet affecté au critique, il ne s'incarne cette fois-ci dans aucun nom et reste un «modèle » abstrait et construit que la « République des lettres » doit chercher à produire. Il faut dire que la figure du critique synthétise ici les qualités du pédagogue et celles de l'orateur chrétiens, dotés, donc, d'un génie universel, et que cela fait incontestablement beaucoup. Reste à savoir si ce n'est qu'idéalement que le lecteur peut être guidé contre les « préjugés » de toute sorte ou s'il y a possibilité réelle de l'armer à ce sujet.

74 Ce sont les termes employés par Baillet, op. cit., p. 20.

75 Le critique est en effet exposé à des dangers tant intérieurs à lui-même (l'excès dans la sévérité ou dans la complaisance par exemple) qu'extérieurs (la haine, le mépris ou la risée de ses adversaires). Baillet s'appuie notamment sur des propos de Marolles et Huet pour décrire la difficulté du métier de critique (op. cit., p. 19).

76 Op. cit., p. 22.

77 Sur cette question, voir J. Lecointe, L'Idéal et la différence. La perception de la personnalité littéraire à la Renaissance, Genève, Droz, 1993, p. 162-172 et 177-181. 


\section{Du « sentiment » du savant au discernement du lecteur}

Les Jugements des savants : un renoncement ?

Il s'agit en effet d'intervenir efficacement pour lutter contre les préjugés « suivant lesquels on a coûtume de juger des Livres ${ }^{78}$ »: Baillet se donne pour mission de former le lecteur. En s'inscrivant une fois encore dans la tradition cartésienne revue par Malebranche et par Port-Royal, il s'en prend notamment à ces « impressions » qui subsistent des jugements sur les livres émis par des maîtres ou par toute autorité auxquels on est trop soumis, et qui oblitèrent le discernement, du moins jusqu'à ce que l'on soit capable d'« étudier seul, et de se rendre le juge de ses propres Maitres ${ }^{79} »$. Ce soubassement théorique favorise des développements longs et circonstanciés sur un grand nombre de «préjugés ", visant à rendre le lecteur plus libre de former des "jugemens nouveaux ${ }^{80} »$. Baillet cherche ainsi à amoindrir les divers écrans s'interposant entre celui-ci et les textes, et donc à éveiller son esprit critique, non seulement à l'égard des «Anciens » (il suit strictement Malebranche pour dénoncer le culte aveugle qui en est fait ${ }^{81}$ ), mais encore à l'égard de nombreux nouveaux aspects, très concrets, de la vie littéraire ${ }^{82}$ : le bibliothécaire profite ici de l'importance croissante désormais accordée au «contexte» dans la production des idées et des ouvrages ${ }^{83}$ pour dénoncer tout ce qui peut conditionner la lecture.

Il faut remarquer qu'à cette étape de son entreprise, après s'en être pourtant pris à différents types précis de critiques dans la première partie de son ouvrage, Baillet indifférencie son public et entend généraliser la portée de son propos. Contrairement à d'autres ouvrages de bibliographie, d'histoire ou de critique littéraires qui, à la même époque, semblent écrits pour un lectorat déterminéé, il ne vise manifestement plus ici un groupe quelconque : les "préjugés » qu'il critique peuvent être ceux de tous, selon les cas - et sans doute cela a-t-il aussi

78 A. Baillet, op. cit., p. 40.

79 Ibid. À son tour, Baillet constate la force de ces «premières impressions » reçues dans l'enfance au travers de l'éducation et des premières études : ibid., p. 73-74.

80 Op. cit., p. 41.

81 Op. cit., p. 41-50.

82 Voir les exemples donnés supra, n. 4.

83 Baillet hérite en effet, notamment, des évolutions de la tradition critique savante qui porte depuis le tournant des XVI e et XVII e siècles plus grande attention au « contexte » de production des textes : voir par ex. J. Céard, «Les transformations du genre du commentaire », art. cit., p. 111. Cependant il profite également des réflexions de Malebranche quant au poids des différentes manières de vivre des hommes (liées à la diversité des conditions, des emplois, des études, des passions, des qualités, etc.) sur leurs idées - et donc, pour Baillet, sur leurs écrits. Voir Malebranche, op. cit., 1. II, II e partie, chap. 2-3, p. 209-210 ; Baillet, op. cit., p. 73.

84 Ne donnons qu'un seul exemple : dans son Recueil des plus belles pièces des Poètes français, tant Anciens que Modernes (1692) et dans son Histoire du théâtre français rédigée dans la dernière décennie du siècle (publ. 1742), Fontenelle cherche manifestement à former un public mondain, tout à la « galanterie ». 
joué un rôle dans la réception de son ouvrage. Cela ne l'empêche pourtant pas de sérier les capacités à juger de différents types de public, et donc d'accepter pragmatiquement la possibilité de spécialités critiques selon les domaines de compétence ou selon des proximités particulières, même s'il est vrai, remarquet-il, qu'aucun livre ne relève réellement que d'un seul savoir ${ }^{55}$. Baillet tend donc ponctuellement à passer de la composition d'une figure idéale du critique à la reconnaissance de sa fragmentation nécessaire dans le réel : concédant en effet notamment qu'il est «aujourd'hui » impossible de tout connaitre, il en vient concrètement à réduire ses exigences, pour le critique, en matière de savoir86. Ge renoncement à l'idéal encyclopédique qu'il fait après tant d'autres prend ainsi acte de la réorganisation du paysage des savoirs en train de s'effectuer.

La rédaction du traité procède donc aussi de la conscience qu'a Baillet que le critique dont il trace les contours ne peut exister. Cet idéal qu'il construit reste un horizon vers lequel tendre et qu'il offre à ses lecteurs comme repère pour qu'ils s'orientent mieux parmi les censeurs d'ouvrages. Sans doute cette figure est-elle aussi la projection d'un idéal de lui-même dans son activité de jugement des livres mais, quoi qu'il en soit, elle est féconde : à défaut de l'existence d'une œuvre totalisante d'un seul et infaillible savant, qui eût exercé son « discernement» dans une « Critique judicieuse » comprenant « tous les Arts et toutes les Sciences », Baillet prépare l'idée d'un nécessaire recueil de tous les jugements produits par " divers Critiques particuliers » sur les "principaux Ouvrages des Auteurs les plus connus ${ }^{87}$ ». C'est donc dans un mouvement d'adaptation au réel que semble s'inscrire et se définir le projet des Fugements des savants. Or cet ouvrage lui offre notamment la possibilité de conférer à la critique - en acte et plus seulement en principe comme il le fait dans le traité introductif - la relative unité disciplinaire qu'il souhaite manifestement lui voir conserver, contrairement aux clivages qu'il peut observer : la « compilation $^{88}$ » large et accueillante de jugements épars réunit de fait sous un même chef de fort différents types de discours 89 . En outre ce rassemblement de critiques "particuliers », palliant la carence d'un censeur universel, lui permet de

85 Baillet s'appuie ici sur Gerson : op. cit., p. 24 ; voir aussi p. 55. Il estime que certaines proximités (de pays, de profession, de société ou d'institut) peuvent contribuer aux jugements de manière avantageuse - dans les limites toutefois imposées par la puissance de l'amourpropre (p. 38).

86 «Quoiqu’il soit donc encore plus nécessaire pour un parfait Critique que pour le parfait Orateur, dont Ciceron étoit si fort en peine, qu'il sache toutes choses et qu'il les sache dans la derniére exactitude ; on n'ose pas néanmoins tant exiger aujourd'hui, parce que ce seroit se mettre hors d'état d'en pouvoir jamais trouver aucun, et se réduire à la nécessité de rejetter toutes sortes de jugemens et de censures, sous prétexte que leurs Auteurs ne peuvent pas être parfaits Critiques dans cette suposition » (ibid., p. 24).

87 Ibid., p. XII (« Avertissement »).

88 Ibid.

89 On y trouve par exemple tant des jugements de goût de certains mondains que des propos de la philologie la plus spécialisée. Voir supra, n. 64, ce que l’on peut constater à ce sujet dans les propos plus théoriques du traité préliminaire. 
continuer à présenter la critique comme une activité susceptible de concerner tous les domaines du savoir, ainsi que le montre le plan encyclopédique initialement prévu pour les Fugements des savants ${ }^{90}$. Cet ouvrage apparaît donc finalement moins comme une véritable et regrettable concession au réel qu'au contraire comme la possibilité de mettre en acte les principes contenus dans le traité préliminaire - ces deux productions constituent bien les deux versants, théorique et pratique, d'une même intention.

\section{Pour un lecteur "Censeur des Critiques"}

Il reste que ce recueil, aux yeux de Baillet, est nécessairement composé de propos aléatoires et contradictoires. Il estime en effet que, malgré le titre qu'il a retenu, les «jugemens » rendus par les divers « savans », bien loin d'être des « arrêts », ne sont le plus souvent que des «Sentimens ou des Opinions ${ }^{91}$ »: l'emploi du terme de « sentiment », en opposition ${ }^{92}$ à celui de «jugement » et à ses connotations juridiques, signale manifestement ici un amoindrissement de l'objectivité et de l'universalité attendues. Il renvoie à l'« opinion » d'un particulier, nécessairement suspecte de contingence et de partialité ou, en tout cas, issue de la différence des esprits, « des humeurs et des goûts ${ }^{93}$ ». Pourtant cette inévitable subjectivité du critique peut être compensée, au moins en partie, par la confrontation systématique des divers jugements émis sur chaque auteur considéré. Car c’est justement cette confrontation elle-même qui permet de passer de la simple communication d'un «sentiment » à un véritable espace de jugement : il ne s'agit donc pas de procéder en amont à une sélection de ces opinions, mais bien plutôt de laisser au lecteur, lui-même sujet à la diversité des goûts, des lumières et des engagements, «le plaisir de ce discernement ${ }^{94}$ ». Cette « compilation » qui va constituer les Fugements des savants entend ainsi introduire le lecteur dans une dimension supérieure : celle de la formation et du discernement personnels. En effet, « chacun des Lecteurs en particulier » sera libre de

se faire impunément le Censeur de ces Critiques avec autant d'autorité que ceux-ci en ont pris pour censurer les autres, ou de se mettre de leur nombre ajoutant son sentiment au leur. 95

90 Voir ce plan (publ. 1694) dans le même volume, op. cit., p. XLIV-LXX. Au début des polémiques concernant Baillet, des bruits avaient couru selon lesquels le premier président de Lamoignon (Guillaume, mort en 1677) aurait été à l'origine de l'architecture générale des Jugements des savants (A. Adam, Histoire de la littérature française au XVII siècle, Paris, Albin Michel, 1997, t. III, p. 455). À notre connaissance, personne ne s'est davantage penché sur cette question.

91 Nous synthétisons ici deux paragraphes de Baillet (op. cit., «Avertissement », p. XIII et XVII).

92 Cette opposition en matière de critique littéraire est commune. On la trouve notamment chez Chapelain dans une lettre à Balzac de 1638, citée dans J.-M. Civardi, op. cit., p. 913.

93 A. Baillet, op. cit., "Avertissement », p. XIV et XIX.

94 Ibid.

95 Op. cit., p. XIII. 
Confronté à la diversité des opinions, le lecteur est invité à faire preuve de discernement : il doit donc devenir actif et adulte au sein de ce nouvel espace qui vise à le rendre, cette fois-ci, lui-même " critique ».

Quel est donc alors le rôle de l'auteur de ce rassemblement critique? Théoriquement le plus discret possible, il reste pourtant un guide, car si sa position semble celle du retrait, elle est aussi celle du surplomb, et sa présence, très assurée et très forte, apparaît finalement comme une version quintessenciée de l'idéal édifié dans le traité : celui qui construit et organise ses notices sait notoirement où il va. Non seulement, en effet, malgré ses déclarations de neutralité ${ }^{96}$, Baillet assume parfois de livrer clairement sa pensée. Il explique par exemple qu'il lui arrive d'« insinuer» ce qu'il pense de l'autorité de ses « garans » et établit dans la foulée une hiérarchie entre des critiques célèbres ${ }^{97}$; mais surtout il donne à voir son ambition dans l'orchestration en sous-main des notices : leur structure même, les enchaînements entre leurs parties, les remarques incidentes, la recherche d'une caractérisation (des genres, des textes, des génies, des évolutions par exemple), les propos de généralisation fondés notoirement sur des principes esthétiques, moraux ou religieux, etc., montrent avec évidence que Baillet cherche à orienter et structurer le jugement du lecteur. Son objectif est évidemment bien moins de dresser un simple répertoire de jugements, que de constituer une critique des critiques.

Après le traité visant à permettre au lecteur de distinguer bons et mauvais censeurs et à déconstruire ses "préoccupations », Baillet entend donc donner accès à un «art » général qui se construit en pratique. Il espère en effet «qu'on pourra trouver dans ce qu'[il] $\operatorname{dir}[\mathrm{a}]$ en rapportant les Jugemens » sur divers auteurs «les Régles et les Maximes » de chacun des domaines concernés : «Ainsi ce sera une espéce d'Art, dont les préceptes consisteront dans les éxemples qui pourront n'être pas moins utiles qu'agréables ${ }^{98}$. » Il n'est donc plus question ici d'une critique prescriptive, fondée sur la production de diverses règles censées gouverner directement tant la création littéraire que l'appréciation des œuvres $^{99}$. Ce n'est que dans la mise en présence d'exemples,

96 Voir par exemple op. cit., p. XIV et XIX.

97 Baillet dit faire une distinction «par éxemple, entre le jugement de Photius et celui du jeune du Verdier, entre ceux de Ciceron, de Denys d'Halicarnasse, de Quintilien, de Longin, et ceux de Sigonius, de Keckerman, de Bodin, de Possevin, etc. » (ibid., p. XIV). Cette liste est à compléter par la sélection des critiques français du siècle précédent faite dans la seconde partie du traité (p. 110) et par les différentes notices des «Critiques" dans les fugements des savants. L'étude de ces jugements portés par Baillet reste à faire, notamment en ce qui concerne les critiques protestants (nous sommes en 1685).

98 «Avertissement», op. cit., p. XXI. Les domaines de connaissance qu'il cite sont « la bonne critique », la grammaire, la traduction, la poésie, l'éloquence, l'histoire, « etc.». L'énumération suit le plan qu'il prévoit.

99 Ainsi classe-t-il Aristote et Horace, pourtant « excellens Critiques », avec ceux qui ont traité de l'art poétique car, estime-t-il, celui qu'ils ont produit « consiste plus en Preceptes qu'en jugemens » (op. cit., I⿳亠丷厂 ${ }^{\text {ere }}$ partie, chap. 5, vol. I, t. II, p. 58). 
orchestrés cependant selon des « raisons » plus ou moins souterraines, que le lecteur est guidé dans la diversité des jugements, des modes de pensée et des goûts, et qu'il est invité à exercer sa propre raison. Ainsi est-ce empiriquement qu'il devrait être formé à l'art de critiquer: Baillet confère au recueil systématique des jugements critiques l'ambition d'une nouvelle discipline, susceptible de produire, d'une nouvelle manière, une nouvelle compétence.

Le travail de Baillet s'inscrit donc dans le profond bouleversement du paysage des savoirs qui a suivi, notamment, la grande secousse cartésienne et les réflexions qu'elle a pu ensuite provoquer sur les fondements de la connaissance. En cherchant à redéfinir l'activité consistant à juger les ouvrages de l'esprit, le bibliothécaire prend acte de l'ébranlement des missions que s'était données la philologie et d'un incontestable discrédit jeté sur certaines évolutions de la « critique » humaniste du siècle précédent et sur le personnage du «censeur », qu'il juge du reste lui-même trop faillible. Pour autant, il n'entend pas laisser la voie libre aux auteurs faibles et « engagés » dont les ouvrages se multiplient, ni aux jugements de goût qui, dorénavant prônés par le public mondain, semblent échapper à toute juridiction. Effectuant un compromis susceptible de doter de nouveaux fondements une discipline d'évaluation de l'ensemble de la production livresque, il prend appui sur les cadres conceptuels d'inspiration cartésienne de ses amis de Port-Royal et de Malebranche pour promouvoir, notamment, l'exercice du jugement, et il prolonge également les réflexions d'un Lamy pour faire dorénavant du critique un guide susceptible, grâce à son discernement, de saisir le «stile » et les «maniéres d'écrire » de tout auteur. Ainsi, dans un contexte où l'esprit de méthode commence à s'appliquer à des domaines autres que les sciences exactes, il en vient à affirmer la pertinence et la nécessité de l'exercice de la raison, secondé toutefois par le savoir le plus vaste, dans le jugement des ouvrages, et le complète par les qualités éthiques du critique, transposées de celles qu'on attend du pédagogue et de l'orateur chrétiens, et qui seules lui permettront d'emporter les suffrages.

Tant d'exigences font de la figure du critique un idéal qui ne peut s'incarner, ce qui permet à Baillet de légitimer le projet de ses fugements des savants. Faute de ce modèle en effet, il lui semble nécessaire non seulement d'éveiller l'esprit critique du lecteur envers les censeurs illégitimes et les «préjugés » qui conditionnent la réception des livres - travail entrepris dans le traité - mais encore de compenser la subjectivité et la contingence des « sentiments » de chacun des juges par leur collation systématique et organisée - travail entrepris dans les fugements des savants. Baillet cherche donc finalement à former le lecteur, et il rejoint en cela des entreprises procédant pourtant de pensées bien 
différentes, comme celle, notamment, de Fontenelle ${ }^{100}$. Le bibliothécaire espère pour sa part ouvrir un véritable espace de jugement dans lequel le lecteur aura à exercer son discernement et à forger son propre "sentiment", orienté s'il le souhaite selon les principes souterrains méthodiquement et savamment orchestrés par celui qui, les ayant jaugés et éprouvés, peut en transmettre l' « intelligence ». Il n'est donc plus question, dans ces deux versants d'une même œuvre, d'édifier une quelconque critique prescriptive, mais bien de former empiriquement l'esprit du lecteur à la «critique » des critiques.

On comprend alors que ce recueil ait été fort bien accueilli par un Bayle, pas uniquement parce que Baillet lui avait consacré une notice élogieuse ${ }^{101}$ et malgré tout ce qui pouvait séparer les deux hommes. Et l'on comprend aussi combien cet élargissement effectué par Baillet de la «critique » savante à l'examen raisonné des livres, élargissement qui est bien moins une disqualification de celle-là que son association à celui-ci, peut unifier en profondeur le projet des Fugements des savants et celui qui portera, une quinzaine d'années plus tard, Les Vies des saints, autre entreprise d' " examen critique ». Enfin, cet élargissement participe aussi sans doute du succès du recueil de Baillet au siècle des Lumières, car ce sont non seulement ses citations et ses propres commentaires, mais encore ses diverses perceptions et représentations de l'univers des ouvrages et de leur jugement qui y sont abondamment utilisés : c'est le cas notamment de la classification justifiée des critiques établie dans les Fugements des savants, qui structure et nourrit encore une large partie de l'article de l'Encyclopédie sur le substantif masculin du mot « Critique ${ }^{102}$ ».

\section{Emmanuelle Mortgat-Longuet} Université Paris Ouest Nanterre

100 Nous nous permettons de renvoyer à notre article « Fontenelle et l'écriture de l'histoire des lettres françaises », Revue Fontenelle, n 6-7, 2008-2009, p. 159-177.

101 On trouvera des précisions sur cet accueil dans L. J. Wang, art. cit., p. 100-101.

102 A. Baillet, op. cit., vol. I, t. II, p. A et N. C'est l'abbé Mallet qui a rédigé l'article « Critique, s. m. » de l'Encyclopédie, auquel servent de fondements le découpage et les propos de Baillet (supra, n. 39 et 62), mais le choix des noms de critiques n'incombe qu'à Mallet. Les Jugements sont cités plusieurs fois dans l'Encyclopédie («Abrégé », « Anonyme », « Livre », etc.). Nous remercions vivement notre collègue Alain Sandrier de nous avoir fait profiter de sa connaissance des Vies des saints et de la critique des Écritures saintes au XVIII siècle. 